\title{
Editorial
}

\section{Advances in Functionalized Materials Research 2016}

\author{
Daniela Predoi, ${ }^{1}$ Mikael Motelica-Heino, ${ }^{2}$ Régis Guegan, ${ }^{2}$ and Philippe Le Coustumer ${ }^{3}$ \\ ${ }^{1}$ National Institute of Materials Physics, P.O. Box MG 07, Bucharest, Magurele, Romania \\ ${ }^{2}$ ISTO, UMR 7327 CNRS Université d'Orléans, 1 A rue de la Férollerie, 45071 Orléans Cedex 2, France \\ ${ }^{3}$ Universite Bordeaux, EA 4592 Géoressources \& Environnement, ENSEGID, 1 allée F. Daguin, 33607 Pessac Cedex, France \\ Correspondence should be addressed to Daniela Predoi; dpredoi@gmail.com
}

Received 20 February 2017; Accepted 20 February 2017; Published 8 March 2017

Copyright (C) 2017 Daniela Predoi et al. This is an open access article distributed under the Creative Commons Attribution License, which permits unrestricted use, distribution, and reproduction in any medium, provided the original work is properly cited.

In the last years, due to the rapid progress of technology, new materials at nanometric scale with special properties have become a flourishing field of research in materials science. The unique physicochemical properties of materials induced by various parameters such as mean size, shape, purity, crystallographic structure, and surface can generate effective solutions to challenging environmental and biomedical problems. As a result of this approach a large number of techniques were developed that enable obtaining novel materials at nanometric scale with specific and reproducible properties and parameters. Below will be highlighted studies on promising properties on the applicability of new materials that could lead to innovative applications in the medical field. Therefore, this special issue is focused on expected advances in the area of functionalized materials at nanometric scale. Due to multidisciplinarity of this topic, this special issue is comprised of a wide range of original research articles as well as review papers on the design and synthesis of functionalized nanomaterials, their structural, morphological, and biological characterization, and their potential uses in medical and environmental applications.

H. Mendoza-Nava et al. reported a study introducing ${ }^{177}$ Lu-labeled nanoparticles conjugated to biomolecules as a new class of theranostic radiopharmaceuticals. In their paper, Mendoza-Nava et al. described the synthesis of ${ }^{177} \mathrm{Lu}-$ Dendrimer- (PAMAM-G4-) Folate-Bombesin with gold nanoparticles (AuNPs) in the dendritic cavity and evaluated their potential use in targeted radiotherapy as well as in the simultaneous detection of folate receptors (FRs) and gastrin-releasing peptide receptors (GRPRs) overexpressed in breast cancer cells. Their luminescence results emphasized that the presence of AuNPs in the conjugate increased the fluorescence intensity, thus creating a multifunctional system suitable for being used as an optical and nuclear imaging agent in targeted radiotherapy and for breast tumors overexpressing GRPRs and FRs.

In their study, N. T. Vo et al. present the conjugation of E. coli O157:H7 antibody on highly luminescent CdSe/ZnS core/shell structures. The authors reported the synthesis of CdSe quantum dots by a chemical green method and their conjugation using E. coli O157:H7 antibody. The FTIR, Zeta potential, TEM, and PL investigations presented in their study have revealed the successful substitution and bioconjugation of E. coli O157:H7 antibody on CdSe/ZnS core/shell structures. The results presented by N. T. Vo et al. offered a new approach in applications for the detection of different bacterium.

C. L. Popa et al. reported the synthesis and physicochemical and biological properties of zinc doped hydroxyapatite (ZnHAp) nanoparticles and their potential use in biological applications. The results presented by the authors showed that the synthesized ZnHAp nanoparticles have slightly elongated morphologies with average diameters between $25 \mathrm{~nm}$ and $18 \mathrm{~nm}$ and a uniform and homogeneous distribution of the constituent elements. Furthermore, the cytotoxicity assay of the studied samples on both E. coli and HepG2 cells revealed that the toxicity of the particles was size dependent. The study underlined that zinc doped hydroxyapatite nanoparticles could be promising candidates for various biomedical applications.

In their study R. Sánchez-Sánchez et al. describe the development of a Collagen Type 1/Chitosan/Dexamethasone 
hydrogel and its anti-inflammatory activity. The results presented by the authors in this study highlighted that human adipose-derived mesenchymal stem cells (hADMSC) can be cultured in the hydrogel without having affected their viability or their proliferation. Furthermore, the results showed that hADMSC secrete the anti-inflammatory cytokine Interleukin-10 (IL-10) but not the inflammatory cytokine tumor necrosis factor-alpha (TNF- $\alpha$ ).

The review conducted by B. G. Kim features various methods such as nonthermal atmospheric pressure plasma, ultraviolet, low level of laser therapy, of surface functionalization of Ti dental implant using nanotopography. The authors acknowledge on-site surface functionalization as a promising strategy for osseointegration enhancement, stating that, in the future, immune or osteoinductive-suppressed patients with diseases such as diabetes mellitus and other genetic disorders could benefit from the promising biological effects of using on-site surface functionalized Ti dental implants.

$S$. Huang et al. reported the use of poly(1-amino-5chloroanthraquinone) (PACA) nanofibrils as novel nanoadsorbents for highly toxic mercury removal from aqueous solutions. The authors described the obtaining of PACA nanofibrils by one-pot synthesis method. Their results showed that PACA nanofibrils were highly recyclable and had a great affinity towards $\mathrm{Hg}$ (II) ions. Furthermore, they have demonstrated that PACA could be used more than 5 times in adsorption experiments without losing their efficiency in removing $\mathrm{Hg}$ (II) from aqueous solutions making them low cost and sustainable adsorbents for water purification.

In their paper S. L. Iconaru et al. reported the obtaining of Ag:HAp-PDMS composite layers by thermal evaporation technique and their structural, morphological, and biological properties. The SEM and EDS studies presented by the authors proved that the Ag:HAp-PDMS composite layers are homogeneous and continuous and present the chemical elements and structure of the Ag:HAp-PDMS coating. Furthermore, the authors demonstrated that Ag:HAp-PDMS composite layers exhibited great antimicrobial properties against S. aureus 0364, E. coli ATCC 25922, and C. albicans 10231 microbial strains suggesting that they can be suitable for being used as antimicrobial coatings.

$\mathrm{X}$. Cao et al. described the synthesis of bentonite supported $\left(\mathrm{N} / \mathrm{Fe}\right.$ ) comodified $\mathrm{TiO}_{2}$ nanoparticles composite (B$\mathrm{N} / \mathrm{Fe}-\mathrm{TiO}_{2}$ ) using a sol-gel method. They have demonstrated that bentonite significantly enhanced the dispersion of $\mathrm{TiO}_{2}$ nanoparticles and increased the specific surface area of the catalysts. The authors have also highlighted that B-N/Fe$\mathrm{TiO}_{2}$ presented an increased photocatalytic efficiency for contaminant degradation under visible light.

C.-C. Negrila et al. presented a complex study regarding the depth chemical composition profile analysis of AuGeNi layer on cleaved n-GaAs (110) using in situ XPS combined with Argon ion sputtering techniques. The authors showed that gold, germanium, and nickel were uniformly distributed in the metallic layer. The XPS measurements revealed that both $\mathrm{Ga}$ and As diffuse to the surface, Ga diffusion being the most pronounced. The formation of Au-Ga alloy has also been shown, indicating a good impact on the quality of ohmic contacts.
In their study N. T. Vo et al. present the synthesis and functionalization of CdSe/ZnS core/shell quantum dots by replacing the phosphine group of the TOP molecule with the carboxyl group of MPA and MSA. The authors have emphasized the substantial enhancement of the luminescence efficiency of CdSe/ZnS compared to that of the CdSe core and have also put into evidence the high stability of CdSe/ZnS-MPA and CdSe/ZnS-MSA using zeta potential measurements. Their results brought relevant information for conjugating biological agents with quantum dots for biomedical applications.

In their work, U. Cottino et al. present a review which highlights the use of tantalum in revision total hip replacement. The authors have emphasized that the osteoinductive properties of tantalum are of great importance on the long term in hip replacements. Moreover, they concluded that tantalum provides a good and reliable substitute of bone, proving that it is a very effective material in orthopedic surgery, especially in revision surgery. Furthermore, the authors have evidence that tantalum's properties such as great ductility, biocompatibility, osteoinductivity, high porosity, elasticity, and bioactivity make this material the best choice for orthopedic implants.

$\mathrm{N}$. Ma et al. in their study present the obtaining of nanostructured WC-Co/Al using WC-12Co powder and pure $\mathrm{Al}$ powder by mechanical alloying (MA). The authors highlighted that ball milling time played an important role in the average WC grain size. On the other hand, N. Ma et al. showed in their study that, using the same spraying parameters, ball-milled WC-Co/Al powders were easier to spread around than the initial WC-12Co powders and that the porosity of the $\mathrm{WC}-\mathrm{Co} / \mathrm{Al}$ coating decreased compared to that of the WC-12Co coating.

S. Miyake et al. studied the improvement of the friction durability of magnetic head-disk interfaces using lubricant films. The authors presented a nanowear and viscoelastic evaluation of the lubricant films on magnetic disk to obtain information about the retention and replenishment. Furthermore, they have investigated the behavior of the lubricant films by load-increase-and-decrease friction tests. The authors demonstrated that the $\mathrm{A} 20 \mathrm{H}$ and Z-tetraol samples exhibited good durability and that the friction coefficients of the $\mathrm{A} 20 \mathrm{H}$-coated disk were stable and exhibited superior friction durability.

\section{Acknowledgments}

The editors would like to thank all the authors who have submitted a manuscript to this special issue. The lead editor would also like to thank all the editors for their time spent in reviewing and assigning reviewers for the submitted manuscripts. Furthermore, the editors thank all the reviewers.

Daniela Predoi Mikael Motelica-Heino Régis Guegan Philippe Le Coustumer 

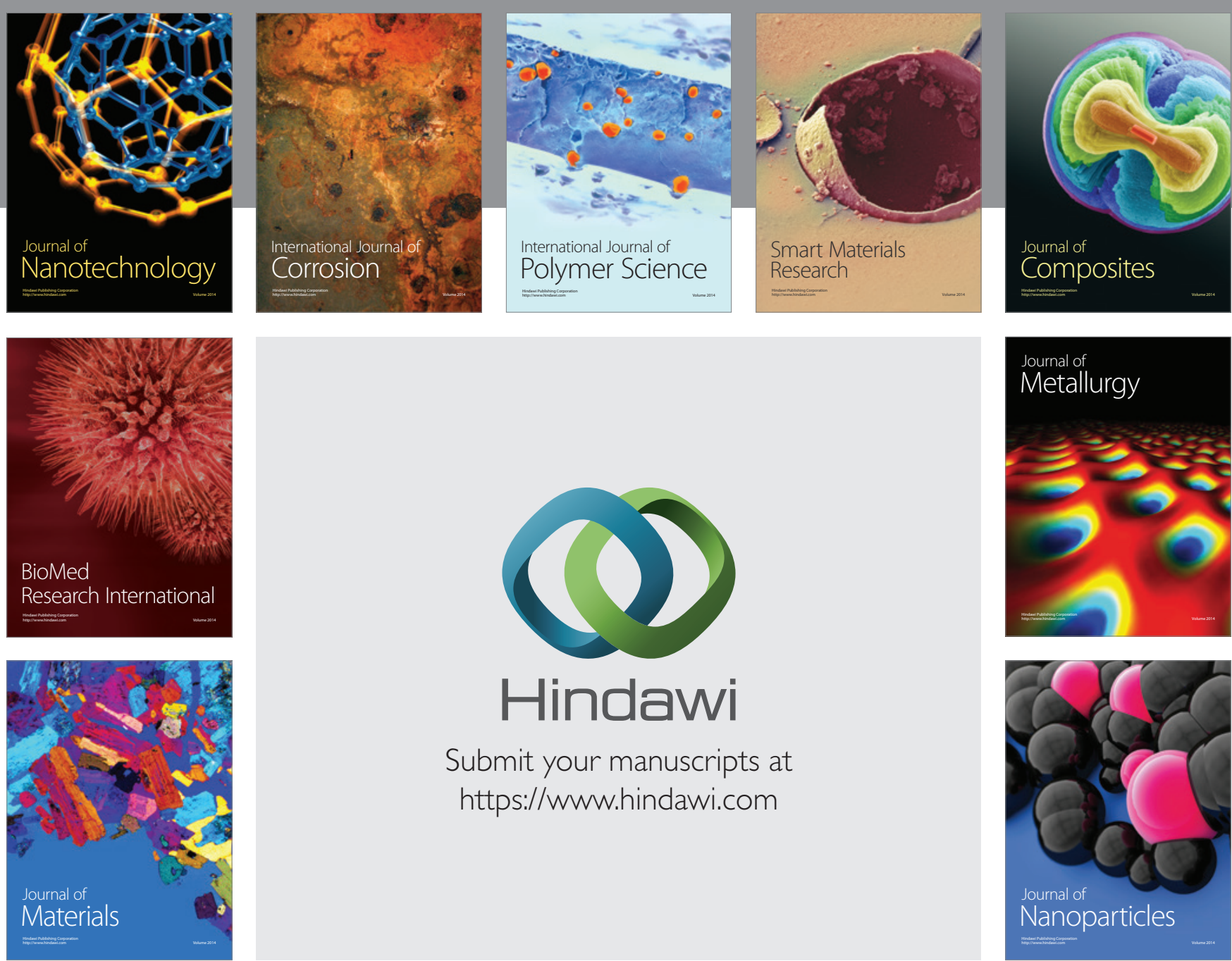

\section{Hindawi}

Submit your manuscripts at

https://www.hindawi.com

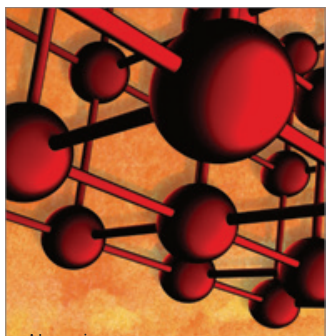

Materials Science and Engineering
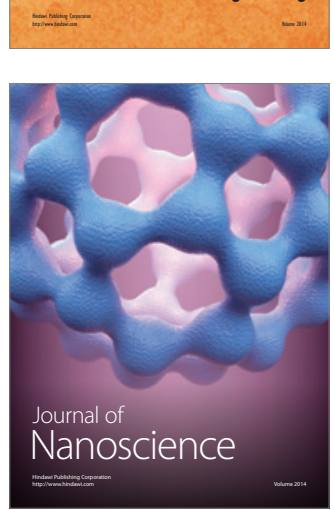
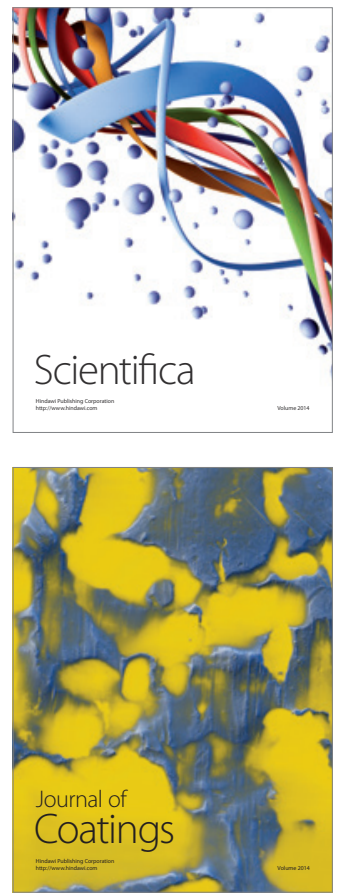
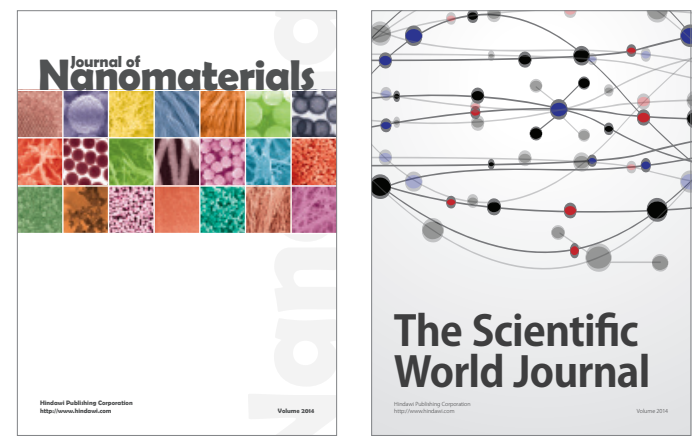

The Scientific World Journal
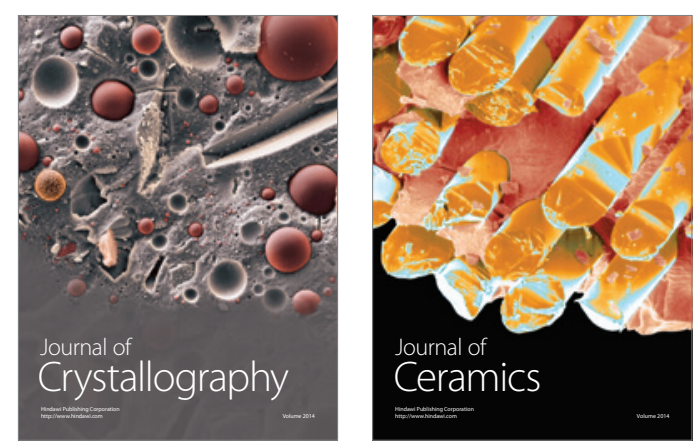
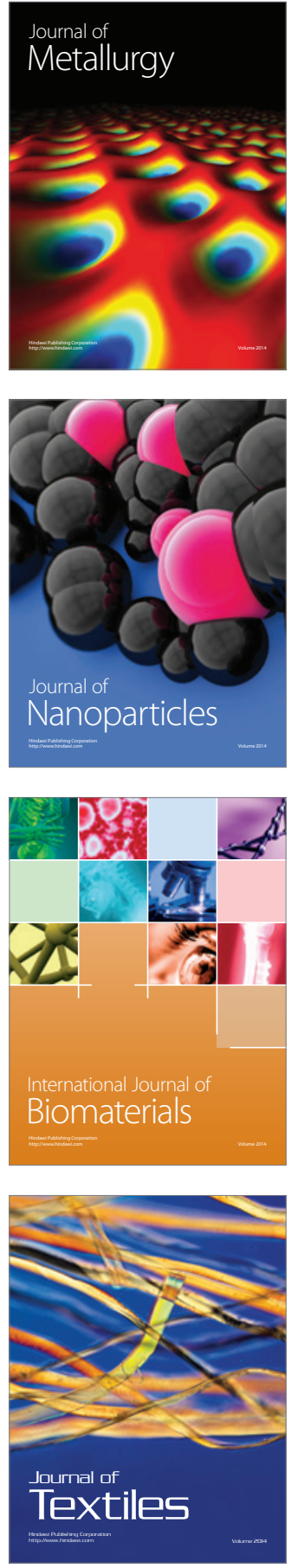